\title{
Karhunen-Loève Expansion for the Second Order Detrended Brownian Motion
}

\author{
Yongchun Zhou, ${ }^{1}$ Xiaohui Ai, ${ }^{2}$ Minghao Lv, ${ }^{1}$ and Boping Tian ${ }^{1}$ \\ ${ }^{1}$ Department of Mathematics, Harbin Institute of Technology, Harbin 150001, China \\ ${ }^{2}$ Department of Mathematics, Northeast Forestry University, Harbin 150040, China \\ Correspondence should be addressed to Boping Tian; bopingt361147@hit.edu.cn
}

Received 8 March 2014; Revised 29 May 2014; Accepted 28 June 2014; Published 16 July 2014

Academic Editor: Salvador Romaguera

Copyright (c) 2014 Yongchun Zhou et al. This is an open access article distributed under the Creative Commons Attribution License, which permits unrestricted use, distribution, and reproduction in any medium, provided the original work is properly cited.

Based on the norm in the Hilbert Space $L^{2}[0,1]$, the second order detrended Brownian motion is defined as the orthogonal component of projection of the standard Brownian motion into the space spanned by nonlinear function subspace. KarhunenLoève expansion for this process is obtained together with the relationship of that of a generalized Brownian bridge. As applications, Laplace transform, large deviation, and small deviation are given.

\section{Introduction}

Let $X=\{X(t), 0 \leq t \leq 1\}$ be a centered and continuous Gaussian process on $[0,1]$ with covariance function

$$
K_{X}(t, s)=E X(t) X(s) .
$$

The Karhunen-Loève expansion of $X$ is given by the (convergent in mean squares) series

$$
X(t)=\sum_{k=1}^{\infty} \eta_{k} \sqrt{\lambda_{k}} f_{k}(t)
$$

where $\left\{\eta_{k}, k \geq 1\right\}$ is a sequence of i.i.d. $N(0,1)$ random variables and $\left\{\lambda_{k}, k \geq 1\right\}$ is at most the countable set of eigenvalues of Fredholm integral operator

$$
T_{X} f(t)=\int_{0}^{1} K_{X}(t, s) f(s) d s
$$

$\left\{f_{k}(t), k \geq 1\right\}$ and forms an orthogonal sequence in $L^{2}[0,1]$ and $\int_{0}^{1} K_{X}(t, t) d t<\infty$.

Deheuvels et al. in [1-4] provided the Karhunen-Loève expansions for the processes that are related with Brownian motion. The Karhunen-Loève expansion for detrended Brownian motion has been studied by $\mathrm{Ai}$ et al. [5]. Note that the detrended Brownian motion in [5] can be viewed as projection to a constant function subspace in $L^{2}[0,1]$. That is,

$$
\int_{0}^{1} \widehat{W}_{1}(t)^{2} d t=\min _{c_{1}, c_{2}} \int_{0}^{1}\left(W(t)-c_{1}-c_{2} t\right)^{2} d t
$$

To generalize the projection idea into nonlinear detrended process, now we consider

$$
\min _{c_{1}, c_{2}, c_{3}} \int_{0}^{1}\left(W(t)-c_{1}-c_{2} t-c_{3} t^{2}\right)^{2} d t
$$

and the optimal constant $c_{j}$ satisfy

$$
\frac{\partial}{\partial c_{j}} \int_{0}^{1}\left(W(t)-c_{1}-c_{2} t-c_{3} t^{2}\right)^{2} d t=0, \quad j=1,2,3 .
$$

It is easy to obtain

$$
\begin{aligned}
& c_{1}=9 \int_{0}^{1} W(s) d s-36 \int_{0}^{1} W(s) s d s+30 \int_{0}^{1} W(s) s^{2} d s, \\
& c_{2}=-36 \int_{0}^{1} W(s) d s+192 \int_{0}^{1} W(s) s d s
\end{aligned}
$$




$$
\begin{gathered}
-180 \int_{0}^{1} W(s) s^{2} d s \\
c_{3}=30 \int_{0}^{1} W(s) d s-180 \int_{0}^{1} W(s) s d s+180 \int_{0}^{1} W(s) s^{2} d s .
\end{gathered}
$$

Let

$$
A=\left(a_{i j}\right)_{3 \times 3}=\left(\begin{array}{ccc}
9 & -36 & 30 \\
-36 & 192 & -180 \\
30 & -180 & 180
\end{array}\right)
$$

we have

$$
c_{j}=\sum_{i=1}^{3} a_{i j} \int_{0}^{1} s^{i-1} W(s) d s, \quad j=1,2,3 .
$$

Now we can define the second order detrended process

$$
\begin{aligned}
\widehat{W}_{2}(t)= & W(t)-\sum_{j=1}^{3} c_{j} t^{j-1} \\
= & W(t)+\left(-9+36 t-30 t^{2}\right) \int_{0}^{1} W(s) d s \\
& +\left(36-192 t+180 t^{2}\right) \int_{0}^{1} W(s) s d s \\
& +\left(-30+180 t-180 t^{2}\right) \int_{0}^{1} W(s) s^{2} d s .
\end{aligned}
$$

\section{Main Results}

We give the following lemma that provides the explicit covariance function.

Lemma 1. For convenience, we add $K_{X}(s, t)$ into formula (11), that is

$$
\begin{aligned}
K_{X}(s, t) & =E\left(\widehat{W}_{2}(t) \widehat{W}_{2}(s)\right) \\
= & t \wedge s-\sum_{p, q=1}^{3} a_{p q}\left(\frac{t}{p}-\frac{t^{p+1}}{p(p+1)}\right) s^{q-1} \\
& -\sum_{i, j=1}^{3} a_{i j}\left(\frac{s}{i}-\frac{s^{i+1}}{i(i+1)}\right) t^{j-1} \\
& +\sum_{p, q=1}^{3} \sum_{i, j=1}^{3} a_{i j} a_{p q} \frac{p+i+2}{(p+1)(p+i+1)(i+1)} t^{j-1} s^{q-1},
\end{aligned}
$$

where $a_{i j}, a_{p q}, i, j, p, q=1,2,3$ is given in (8).
Proof. Consider

$$
\widehat{W}_{2}(t)=W(t)-\sum_{j=1}^{3} c_{j} t^{j-1}, \quad 0 \leq t \leq 1
$$

and $\widehat{W}_{2}(t)$ is a mean zero Gaussian process; we obtain

$$
\begin{aligned}
E\left(\widehat{W}_{2}(t) \widehat{W}_{2}(s)\right) & \\
= & E \widehat{W}_{2}(t) \widehat{W}_{2}(s) \\
= & E\left(W(t)-\sum_{j=1}^{3} c_{j} t^{j-1}\right)\left(W(s)-\sum_{q=1}^{3} c_{q} s^{q-1}\right) \\
= & E\left(W(t)-\sum_{i, j=1}^{3} a_{i j}\left(\int_{0}^{1} u^{i-1} W(u) d u\right) t^{j-1}\right) \\
& \cdot E\left(W(s)-\sum_{p, q=1}^{3} a_{p q}\left(\int_{0}^{1} v^{p-1} W(v) d v\right) s^{q-1}\right) .
\end{aligned}
$$

We notice that

$$
\begin{gathered}
E(W(t) W(s))=t \wedge s \\
E\left(W(t) \int_{0}^{1} v^{p-1} W(v) d v\right) \\
=E\left(\int_{0}^{1} W(t) W(v) v^{p-1} d v\right) \\
=\int_{0}^{1}(t \wedge v) v^{p-1} d v \\
=\int_{0}^{t} v^{p} d v+\int_{t}^{1} t v^{p-1} d v \\
E\left(\int_{0}^{1} u^{i-1} W(u) d u\right)\left(\int_{0}^{1} v^{p-1} W(v) d v\right) \\
=\int_{0}^{1} u^{i-1} E\left(W(u) \int_{0}^{1} v^{p-1} W(v) d v\right) d u \\
=\int_{0}^{1} u^{i-1}\left(\frac{u}{p}-\frac{u^{p+1}}{p(p+1)}\right) d u \\
(p+1)(p+i+1)(i+1)
\end{gathered}
$$

Substituting (16), (17), and (19) into (15), we derive

$$
E\left(\widehat{W}_{2}(t) \widehat{W}_{2}(s)\right)
$$

$$
=t \wedge s-\sum_{p, q=1}^{3} a_{p q}\left(\frac{t}{p}-\frac{t^{p+1}}{p(p+1)}\right) s^{q-1}
$$




$$
\begin{aligned}
& -\sum_{i, j=1}^{3} a_{i j}\left(\frac{s}{i}-\frac{s^{i+1}}{i(i+1)}\right) t^{j-1} \\
& +\sum_{p, q=1}^{3} \sum_{i, j=1}^{3} a_{i j} a_{p q} \frac{p+i+2}{(p+1)(p+i+1)(i+1)} t^{j-1} s^{q-1} .
\end{aligned}
$$

Lemma 2 (see [3]). If $t \in[0,1], \varsigma_{j}(t)=\sum_{k=1}^{\infty} \omega_{k} \sqrt{\lambda_{k, j}} e_{k, j}(t)$, $j=1,2, \ldots$, then the condition

$$
\int_{[0,1]} \varsigma_{1}^{2}(t) d t \stackrel{\operatorname{law}}{=} \int_{[0,1]} \varsigma_{2}^{2}(t) d t
$$

is equivalent to the identity

$$
\lambda_{k, 1}=\lambda_{k, 2} \quad \forall k \geq 1
$$

In the following, we will give some preliminaries, notions, and facts that are needed in Theorem 3. For $v>-1, J_{v}(\cdot)$ is Bessel function [6] with index $v$ and the positive zeros of $J_{v}(\cdot)$ are infinite sequence $0<z_{v, 1}<z_{v, 2}<\cdots$. When $v=3 / 2$, $v=5 / 2$, the positive zeros of $J_{3 / 2, k}, J_{5 / 2, k}$ are $z_{3 / 2, k}, z_{5 / 2, k}, k=$ $1,2, \ldots$, and they are in such a way that

$$
0<z_{3 / 2,1}<z_{5 / 2,1}<z_{3 / 2,2}<\cdots
$$

Now we can state one of the main results of this paper.

Theorem 3. For the second order detrended Brownian motion $\widehat{W}_{2}(t)$ and a generalized Brownian bridge $B_{2}(t)$ with $n=2$ in [7],

$$
\begin{aligned}
B_{2}(t)= & B(t)-\frac{1}{36} t\left(60 t^{2}+18 t-67\right) B(1) \\
& -t\left(60 t^{2}-96 t+11\right) \int_{0}^{1} B(s) d s \\
& +10 t\left(12 t^{2}-18 t+1\right) \int_{0}^{1} B(s) s d s .
\end{aligned}
$$

One has the distribution identities

$$
\begin{aligned}
& \int_{0}^{1} \widehat{W}_{2}(t)^{2} d t \stackrel{\text { law }}{=} \int_{0}^{1} B_{2}(t)^{2} d t \\
& \stackrel{\text { law }}{=} \sum_{k \geq 1} \frac{\eta_{k}^{2}}{4 z_{3 / 2, k}^{2}}+\sum_{k \geq 1} \frac{\eta_{k}^{* 2}}{4 z_{5 / 2, k}^{2}},
\end{aligned}
$$

where $\left\{\eta_{k}, k \geq 1\right\}$ and $\left\{\eta_{k}^{*}, k \geq 1\right\}$ denote two independent sequences of independently and identically distributed $N(0,1)$ random variables.
Proof. By straightforward induction based on the equation and splitting the integration range from $t$, we get

$$
\begin{aligned}
\lambda f(t)= & \int_{0}^{t} s f(s) d s+t \int_{t}^{1} f(s) d s \\
& -\sum_{p, q=1}^{3} a_{p q}\left(\frac{t}{p}-\frac{t^{p+1}}{p(p+1)}\right) \int_{0}^{1} s^{q-1} f(s) d s \\
& -\sum_{i, j=1}^{3} a_{i j} t^{j-1} \int_{0}^{1}\left(\frac{s}{i}-\frac{s^{i+1}}{i(i+1)}\right) f(s) d s \\
& +\sum_{p, q=1}^{3} \sum_{i, j=1}^{3} a_{i j} a_{p q} \frac{p+i+2}{(p+1)(p+i+1)(i+1)} t^{j-1} \\
& \times \int_{0}^{1} s^{q-1} f(s) d s .
\end{aligned}
$$

By differentiation of both sides of (23) with respect to $t$, we have

$$
\begin{aligned}
\lambda f^{\prime}(t)= & \int_{t}^{1} f(s) d s-\sum_{p, q=1}^{3} a_{p q} \frac{1-t^{p}}{p} \int_{0}^{1} s^{q-1} f(s) d s \\
& -\sum_{i=1, j=2}^{3}(j-1) a_{i j} t^{j-2} \int_{0}^{1}\left(\frac{s}{i}-\frac{s^{i+1}}{i(i+1)}\right) f(s) d s \\
& +\sum_{p, q=1}^{3} \sum_{i=1, j=2}^{3} a_{i j} a_{p q} \frac{(p+i+2)(j-1)}{(p+1)(p+i+1)(i+1)} t^{j-2} \\
& \times \int_{0}^{1} s^{q-1} f(s) d s .
\end{aligned}
$$

By differentiation of both sides of (24) with respect to $t$, we have

$$
\begin{aligned}
\lambda f^{\prime \prime}( & t)+f(t) \\
= & \sum_{i, p, q=1}^{3} a_{i 3} a_{p q} \frac{2(p+i+2)}{(p+1)(p+i+1)(i+1)} \int_{0}^{1} s^{q-1} f(s) d s \\
& -2 \sum_{i=1}^{3} a_{i 3} \int_{0}^{1}\left(\frac{s}{i}-\frac{s^{i+1}}{i(i+1)}\right) f(s) d s \\
& +\sum_{q=1}^{3} a_{1 q} \int_{0}^{1} s^{q-1} f(s) d s \\
& +\left(\sum_{q=1}^{3} a_{2 q} \int_{0}^{1} s^{q-1} f(s) d s\right) t \\
& +\left(\sum_{q=1}^{3} a_{3 q} \int_{0}^{1} s^{q-1} f(s) d s\right) t^{2} .
\end{aligned}
$$


We can simplify this equation to

$$
\lambda f^{\prime \prime}(t)+f(t)+b_{1}+b_{2} t+b_{3} t^{2}=0,
$$

where

$$
\begin{aligned}
b_{1}= & -\sum_{i, p, q=1}^{3} a_{i 3} a_{p q} \frac{2(p+i+2)}{(p+1)(p+i+1)(i+1)} \int_{0}^{1} s^{q-1} f(s) d s \\
& +2 \sum_{i=1}^{3} a_{i 3} \int_{0}^{1}\left(\frac{s}{i}-\frac{s^{i+1}}{i(i+1)}\right) f(s) d s \\
& -\sum_{q=1}^{3} a_{1 q} \int_{0}^{1} s^{q-1} f(s) d s \\
b_{2}= & -\sum_{q=1}^{3} a_{2 q} \int_{0}^{1} s^{q-1} f(s) d s \\
b_{3}= & -\sum_{q=1}^{3} a_{3 q} \int_{0}^{1} s^{q-1} f(s) d s .
\end{aligned}
$$

We solve the inhomogeneous second differential equation to obtain

$$
f(t)=c_{1} \cos \frac{t}{\sqrt{\lambda}}+c_{2} \sin \frac{t}{\sqrt{\lambda}}+2 \lambda b_{3}-b_{1}-b_{2} t-b_{3} t^{2}
$$

We substitute $f(t)$ into (28) and (29) to obtain

$$
\begin{aligned}
& \left(\sqrt{\lambda} \sin \frac{1}{\sqrt{\lambda}}+6 \lambda \cos \frac{1}{\sqrt{\lambda}}-12 \lambda \sqrt{\lambda} \sin \frac{1}{\sqrt{\lambda}}+6 \lambda\right) c_{1} \\
& +\left(-\sqrt{\lambda} \cos \frac{1}{\sqrt{\lambda}}+6 \lambda \sin \frac{1}{\sqrt{\lambda}}\right. \\
& \left.\quad+12 \lambda \sqrt{\lambda} \cos \frac{1}{\sqrt{\lambda}}-12 \lambda \sqrt{\lambda}+\sqrt{\lambda}\right) c_{2} \\
& \left.=0, \quad-2 \sqrt{\lambda} \sin \frac{1}{\sqrt{\lambda}}-14 \lambda \cos \frac{1}{\sqrt{\lambda}}+30 \lambda \sqrt{\lambda} \sin \frac{1}{\sqrt{\lambda}}-16 \lambda\right) c_{1} \\
& +\left(2 \sqrt{\lambda} \cos \frac{1}{\sqrt{\lambda}}-14 \lambda \sin \frac{1}{\sqrt{\lambda}}\right. \\
& \left.\quad-30 \lambda \sqrt{\lambda} \cos \frac{1}{\sqrt{\lambda}}+30 \lambda \sqrt{\lambda}-3 \sqrt{\lambda}\right) c_{2}
\end{aligned}
$$$$
=0 \text {. }
$$

In order that there are nonzero choices for $c_{1}, c_{2}$, the determinant of the above two equations has to be zero, which can be written as

$$
D_{11} D_{22}-D_{12} D_{21}=0
$$

where

$$
\begin{aligned}
D_{11}= & \sqrt{\lambda} \sin \frac{1}{\sqrt{\lambda}}+6 \lambda \cos \frac{1}{\sqrt{\lambda}}-12 \lambda \sqrt{\lambda} \sin \frac{1}{\sqrt{\lambda}}+6 \lambda, \\
D_{12}= & -\sqrt{\lambda} \cos \frac{1}{\sqrt{\lambda}}+6 \lambda \sin \frac{1}{\sqrt{\lambda}}+12 \lambda \sqrt{\lambda} \cos \frac{1}{\sqrt{\lambda}} \\
& -12 \lambda \sqrt{\lambda}+\sqrt{\lambda}, \\
D_{21}= & -2 \sqrt{\lambda} \sin \frac{1}{\sqrt{\lambda}}-14 \lambda \cos \frac{1}{\sqrt{\lambda}} \\
& +30 \lambda \sqrt{\lambda} \sin \frac{1}{\sqrt{\lambda}}-16 \lambda, \\
D_{22}= & 2 \sqrt{\lambda} \cos \frac{1}{\sqrt{\lambda}}-14 \lambda \sin \frac{1}{\sqrt{\lambda}}-30 \lambda \sqrt{\lambda} \cos \frac{1}{\sqrt{\lambda}} \\
& +30 \lambda \sqrt{\lambda}-3 \sqrt{\lambda} .
\end{aligned}
$$

We obtain, after some simplification,

$$
\begin{aligned}
& 24 \lambda^{2} \sqrt{\lambda}+4 \lambda \sqrt{\lambda} \\
& =\left(24 \lambda^{2}-\lambda\right) \sin \frac{1}{\sqrt{\lambda}}+\left(24 \lambda^{2} \sqrt{\lambda}-8 \lambda \sqrt{\lambda}\right) \cos \frac{1}{\sqrt{\lambda}} .
\end{aligned}
$$

Then $\lambda \neq 0$ is an eigenvalue if and only if (34) holds. We therefore obtain

$D(\lambda)=-720\left(\left(24 \lambda^{-7 / 2}-\lambda^{-5 / 2}\right) \sin \lambda^{1 / 2}\right.$

$$
\left.+\left(24 \lambda^{-4}-8 \lambda^{-3}\right) \cos \lambda^{1 / 2}-24 \lambda^{-4}-4 \lambda^{-3}\right),
$$

with $D(0)=1$.

According to the trigonometric function formula

$$
\begin{aligned}
& \sin \frac{1}{\sqrt{\lambda}}=2 \sin \frac{1}{2 \sqrt{\lambda}} \cos \frac{1}{2 \sqrt{\lambda}}, \\
& \cos \frac{1}{\sqrt{\lambda}}=2 \cos ^{2} \frac{1}{2 \sqrt{\lambda}}-1=1-2 \sin ^{2} \frac{1}{2 \sqrt{\lambda}},
\end{aligned}
$$

we can observe that

$$
D_{11} D_{22}-D_{12} D_{21}=-12 \pi \sqrt{\lambda} J_{3 / 2}\left(\frac{1}{2 \sqrt{\lambda}}\right) J_{5 / 2}\left(\frac{1}{2 \sqrt{\lambda}}\right)=0 \text {, }
$$

where $J_{3 / 2}(z), J_{5 / 2}(z)$ are Bessel functions as follows:

$$
\begin{aligned}
& J_{3 / 2}(z)=\frac{\sqrt{2 \pi \cdot z}}{\pi}\left(\frac{\sin z}{z^{2}}-\frac{\cos z}{z}\right), \\
& J_{5 / 2}(z)=\frac{\sqrt{2 \pi \cdot z}}{\pi}\left(\left(-\frac{1}{z}+\frac{3}{z^{3}}\right) \sin z-\frac{3}{z^{2}} \cos z\right),
\end{aligned}
$$

which gives two sequences of eigenvalues of (37), namely, $\left(2 z_{3 / 2, k}\right)^{-2}$ and $\left(2 z_{5 / 2, k}\right)^{-2}$. 
Similarly, we can obtain the two eigenvalues $\left(2 z_{3 / 2, k}\right)^{-2}$, $\left(2 z_{5 / 2, k}\right)^{-2}$ corresponding to those of integral operator of a generalized Brownian bridge $B_{2}(t)$. Note that the integral operator is

$$
\int_{0}^{1} K_{2}(s, t) f(s) d s .
$$

Actually, in Lemma 2, we have the distribution identities

$$
\begin{aligned}
& \int_{0}^{1} \widehat{W}_{2}(t)^{2} d t \stackrel{\text { law }}{=} \int_{0}^{1} B_{2}(t)^{2} d t \\
& \stackrel{\text { law }}{=} \sum_{k \geq 1} \frac{\eta_{k}^{2}}{4 z_{3 / 2, k}^{2}}+\sum_{k \geq 1} \frac{\eta_{k}^{* 2}}{4 z_{5 / 2, k}^{2}} .
\end{aligned}
$$

Remark 4. From (11) and (22), we derive that

$$
\begin{aligned}
\int_{0}^{1} K_{X}(t, t) d t & =\int_{0}^{1} E\left(\widehat{W}_{2}(t)^{2}\right) d t=E \int_{0}^{1} \widehat{W}_{2}(t)^{2} d t \\
& =\sum_{k \geq 1} \frac{1}{4 z_{3 / 2, k}^{2}}+\sum_{k \geq 1} \frac{1}{4 z_{5 / 2, k}^{2}} \\
& =\frac{1}{40}+\frac{1}{56}=\frac{3}{140}
\end{aligned}
$$

by using the Rayleigh's formula, for $v=3 / 2$ and $v=5 / 2$ (see, e.g., [3, (1.91), page 77] and [6, page 502]).

To check (41), from (11), we infer that

$$
\begin{aligned}
& \int_{0}^{1} K_{X}(t, t) d t \\
& =\int_{0}^{1}\left[t-\sum_{p, q=1}^{3} a_{p q}\left(\frac{t^{q}}{p}-\frac{t^{p+q}}{p(p+1)}\right)\right. \\
& \quad-\sum_{i, j=1}^{3} a_{i j}\left(\frac{t^{j}}{i}-\frac{t^{i+j}}{i(i+1)}\right) \\
& \quad+\sum_{p, q=1}^{3} \sum_{i, j=1}^{3} a_{i j} a_{p q} \\
& \times \frac{3}{140}
\end{aligned}
$$

which is in agreement with (41).

\section{Applications}

In this section, the relevant applications of Karhunen-Loève expansion are given.
Proposition 5. For each $\theta \in R$, one has

$$
\begin{gathered}
E \exp \left(-\frac{\theta^{2}}{2} \int_{0}^{1} \widehat{W}_{2}(t)^{2} d t\right) \\
=\left\{-720\left(\left(24 \theta^{-7}-\theta^{-5}\right) \sin \theta+\left(-24 \theta^{-8}+8 \theta^{-6}\right) \cos \theta\right.\right. \\
\left.\left.+24 \theta^{-8}+4 \theta^{-6}\right)\right\}^{-1 / 2}
\end{gathered}
$$

Proof.

$$
\begin{aligned}
& E \exp \left(-\frac{\theta^{2}}{2} \int_{0}^{1} \widehat{W}_{2}(t)^{2} d t\right) \\
& =E \exp \left(-\frac{\theta^{2}}{2} \sum_{k=1}^{\infty} \lambda_{k} \xi_{k}^{2}\right) \\
& =\prod_{k=1}^{\infty}\left(1+\lambda_{k} \theta^{2}\right)^{-1 / 2}=\left(D\left(-\theta^{2}\right)\right)^{-1 / 2} \\
& =\left\{-720\left(\left(24 \theta^{-7}-\theta^{-5}\right) \sin \theta+\left(-24 \theta^{-8}+8 \theta^{-6}\right) \cos \theta\right.\right. \\
& \left.\left.\quad+24 \theta^{-8}+4 \theta^{-6}\right)\right\}^{-1 / 2}
\end{aligned}
$$

where $\lambda_{1}>\lambda_{2}>\cdots>0$ and $\sum_{k=1}^{\infty} \lambda_{k}<\infty$.

Proposition 6. If $x>0$, then

$$
\begin{aligned}
& P\left(\int_{0}^{1} \widehat{W}_{2}^{2}(t) d t>x\right) \\
& =\frac{1}{\pi} \sum_{k=1}^{\infty}(-1)^{k+1} \\
& \quad \times \int_{\gamma_{2 k-1}}^{\gamma_{2 k}}\left(e^{-u x / 2}\right. \\
& \quad \times\left(u \left(\mid-720\left(\left(24 u^{-7 / 2}-u^{-5 / 2}\right) \sin u^{1 / 2}\right.\right.\right. \\
& \quad+\left(24 u^{-4}-8 u^{-3}\right) \cos u^{1 / 2} \\
& \left.\left.\left.\left.\quad-24 u^{-4}-4 u^{-3}\right) \mid\right)^{1 / 2}\right)^{-1}\right) d u
\end{aligned}
$$

where $\gamma_{k}=\lambda_{k}^{-1}, k=1,2, \ldots$

Proof. It can be proved by the Smirnov formula $[8,9]$, formula (23), and the definition of the Fredholm determinant. Similar proof method can be found from Proposition 3.3 in [10].

Next, we give the large deviation and small deviation probabilities of the second order detrended Brownian motion with respect to the norm in the Hilbert Space $L^{2}[0,1]$. 
Proposition 7. Consider $x \rightarrow \infty$,

$$
\begin{aligned}
P\left(\int_{0}^{1} \widehat{W}_{2}(t)^{2} d t>x\right) & (1+o(1))\left(\frac{2}{\pi}\right)^{1 / 2}\left(2 z_{3 / 2,1}\right)^{-2} x^{-1 / 2} \exp \left(-2 z_{3 / 2,1}^{2} x\right) \\
= & \left\{7 2 0 \left(\left(-\frac{3}{2^{4}} z_{3 / 2,1}^{-9}+\frac{13}{2^{8}} z_{3 / 2,1}^{-7}\right) \sin 2 z_{3 / 2,1}\right.\right. \\
& +\left(-\frac{3}{2^{5}} z_{3 / 2,1}^{-10}+\frac{9}{2^{6}} z_{3 / 2,1}^{-8}-\frac{1}{2^{7}} z_{3 / 2,1}^{-6}\right) \\
& \left.\left.\times \cos 2 z_{3 / 2,1}+\frac{3}{2^{5}} z_{3 / 2,1}^{-10}+\frac{3}{2^{6}} z_{3 / 2,1}^{-8}\right)\right\}^{-1 / 2}
\end{aligned}
$$

Proof. By Deheuvels [2] and Martynov [8], we have for all $x>$ 0

$$
\begin{aligned}
& P\left(\int_{0}^{1} \widehat{W}_{2}(t)^{2} d t>x\right) \\
& =(1+o(1))\left(\frac{2}{\pi}\right)^{1 / 2} \gamma_{1}^{-1}\left(-D^{\prime}\left(\gamma_{1}\right)\right)^{-1 / 2} \\
& \quad \times x^{-1 / 2} \exp \left(-\frac{\gamma_{1} x}{2}\right)
\end{aligned}
$$

we take $D(\lambda)$ and $\gamma_{1}=\left(2 z_{3 / 2,1}\right)^{2}$ into (47), and then the proof is completed.

Proposition 8. There exists a constant $c>0$ such that

$$
\begin{aligned}
& P\left(\int_{0}^{1} \widehat{W}_{2}(t)^{2} d t \leq \varepsilon\right) \\
& \quad=(c+o(1)) \varepsilon^{-2} \exp \left(-\frac{1}{8 \varepsilon}\right), \quad \text { as } \varepsilon \longrightarrow 0 .
\end{aligned}
$$

Proof. We start with proving (48) by recalling Li, 1992 [11, 12]. Given two sequences $a_{k}>0$ and $b_{k}>0$ with

$$
\sum_{k \geq 1} a_{k}<\infty, \quad \sum_{k \geq 1} b_{k}<\infty, \quad \sum_{k \geq 1}\left|1-\frac{a_{k}}{b_{k}}\right|<\infty,
$$

we have, as $\varepsilon \rightarrow 0$,

$$
\begin{aligned}
& P\left(\sum_{k \geq 1} a_{k} \xi_{k}^{2} \leq \varepsilon\right) \\
& \quad=(1+o(1))\left(\prod_{k \geq 1} \frac{b_{k}}{a_{k}}\right)^{1 / 2} P\left(\sum_{k \geq 1} b_{k} \xi_{k}^{2} \leq \varepsilon\right) .
\end{aligned}
$$

By the asymptotic formula for zeros of Bessel function

$$
\begin{aligned}
z_{3 / 2, k} & =\left(k+\frac{1}{2}\right) \pi+O\left(k^{-1}\right), \quad k \longrightarrow \infty, \\
z_{5 / 2, k} & =(k+1) \pi+O\left(k^{-1}\right), \quad k \longrightarrow \infty,
\end{aligned}
$$

then $a_{k}=\lambda_{k}, b_{2 k-1}=((2 k+1) \pi)^{-2}$, and $b_{2 k}=((2 k+2) \pi)^{-2}$, $k \in N$, which satisfy (49) and by the distribution identity $\int_{0}^{1} \widehat{W}_{2}^{2}(t) d t=\sum_{k \geq 1} \lambda_{2 k-1} \eta_{k}^{2}+\sum_{k \geq 1} \lambda_{2 k} \eta_{2 k}^{* 2}$ and (50), there exists a constant $c_{1}$, such that

$$
\begin{aligned}
P\left(\int_{0}^{1} \widehat{W}_{2}(t)^{2} d t \leq \varepsilon\right) \\
=P\left(\sum_{k \geq 1} \lambda_{2 k-1} \eta_{k}^{2}+\sum_{k \geq 1} \lambda_{2 k} \eta_{k}^{* 2} \leq \varepsilon\right) \\
=(1+o(1)) \prod_{k \geq 1}\left(\frac{b_{k}}{a_{k}}\right)^{1 / 2} P\left(\sum_{k \geq 1} b_{k} \xi_{k}^{2} \leq \varepsilon\right) \\
\left.=(1+o(1)) c_{1}\right) \\
\quad \times P\left(\sum_{k \geq 1} \frac{\xi_{2 k-1}^{2}}{((2 k+1) \pi)^{2}}+\sum_{k \geq 1} \frac{\xi_{2 k}^{2}}{((2 k+2) \pi)^{2}} \leq \varepsilon\right) \\
=(1+o(1)) c_{1} P\left(\sum_{k \geq 1}(k+2)^{-2} \xi_{k}^{2} \leq \varepsilon \pi^{2}\right), \quad \text { as } \varepsilon \longrightarrow 0 .
\end{aligned}
$$

Also, for all $d>-1$, there exists a constant $c_{2}>0$, such that, as $\varepsilon \rightarrow 0$,

$$
P\left(\sum_{k \geq 1}(k+d)^{-2} \xi_{k}^{2} \leq \varepsilon \pi^{2}\right)=(1+o(1)) c_{2} \varepsilon^{-d} \exp \left(-\frac{1}{8 \varepsilon}\right) .
$$

Connecting (52) with (53), we can obtain the proposition.

\section{Conflict of Interests}

The authors declare that there is no conflict of interests regarding the publication of this paper.

\section{Acknowledgments}

This work is supported by the National Natural Science Fund (71350005), Heilong Jiang Province Natural Science Fund (G200815), and the Fundamental Research Funds for the Central Universities (no. DL13BBX10).

\section{References}

[1] P. Deheuvels, G. Peccati, and M. Yor, "On quadratic functionals of the Brownian sheet and related processes," Stochastic Processes and Their Applications, vol. 116, no. 3, pp. 493-538, 2006.

[2] P. Deheuvels, "A Karhunen-Loève expansion for a meancentered Brownian bridge," Statistics and Probability Letters, vol. 77, no. 12, pp. 1190-1200, 2007.

[3] P. Deheuvels and G. V. Martynov, "Karhunen-Loève expansions for weighted Wiener processes and Brownian Bridges via Bessel Functions," Progress in Probability, vol. 55, pp. 57-93, 2003.

[4] P. Deheuvels, "Karhunen-Loève expansions of mean-centered Wiener processes," High Dimensional Probability, vol. 51, pp. 6276, 2006. 
[5] X. Ai, W. V. Li, and G. Liu, "Karhunen-Loeve expansions for the detrended Brownian motion," Statistics \& Probability Letters, vol. 82, no. 7, pp. 1235-1241, 2012.

[6] G. N. Watson, A Treatise on the Theory of Bessel Functions, Cambridge University Press, Cambridge, UK, 1952.

[7] I. B. MacNeill, "Properties of sequences of partial sums of polynomial regression residuals with applications to tests for change of regression at unknown times," The Annals of Statistics, vol. 6, no. 2, pp. 422-433, 1978.

[8] G. V. Martynov, "A generalization of Smirnov's formula for the distribution functions of quadratic forms," Theory of Probability \& Its Applications, vol. 22, no. 3, pp. 614-620, 1977.

[9] N. Smirnov, "Table for estimating the goodness of fit of empirical distributions," Annals of Mathematical Statistics, vol. 19, pp. 279-281, 1948.

[10] M. Barczy and E. Iglói, "Karhunen-Loève expansions of $\alpha$ Wiener bridges," Central European Journal of Mathematics, vol. 9, no. 1, pp. 65-84, 2011.

[11] W. V. Li, "Comparison results for the lower tail of Gaussian seminorms," Journal of Theoretical Probability, vol. 5, no. 1, pp. 1-31, 1992.

[12] W. V. Li, "Limit theorems for the square integral of Brownian motion and its increments," Stochastic Processes and Their Applications, vol. 41, no. 2, pp. 223-239, 1992. 


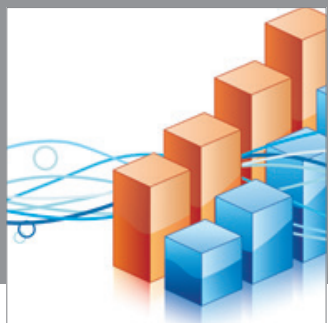

Advances in

Operations Research

mansans

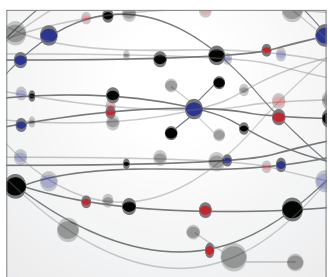

The Scientific World Journal
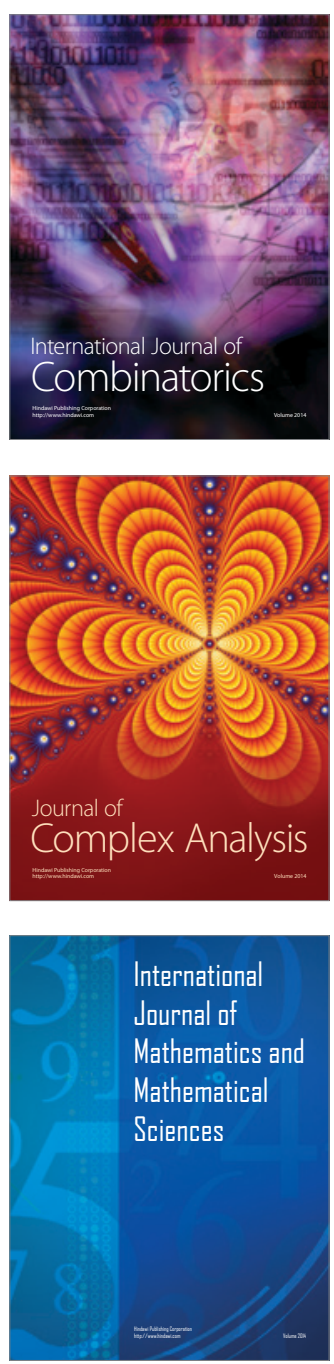
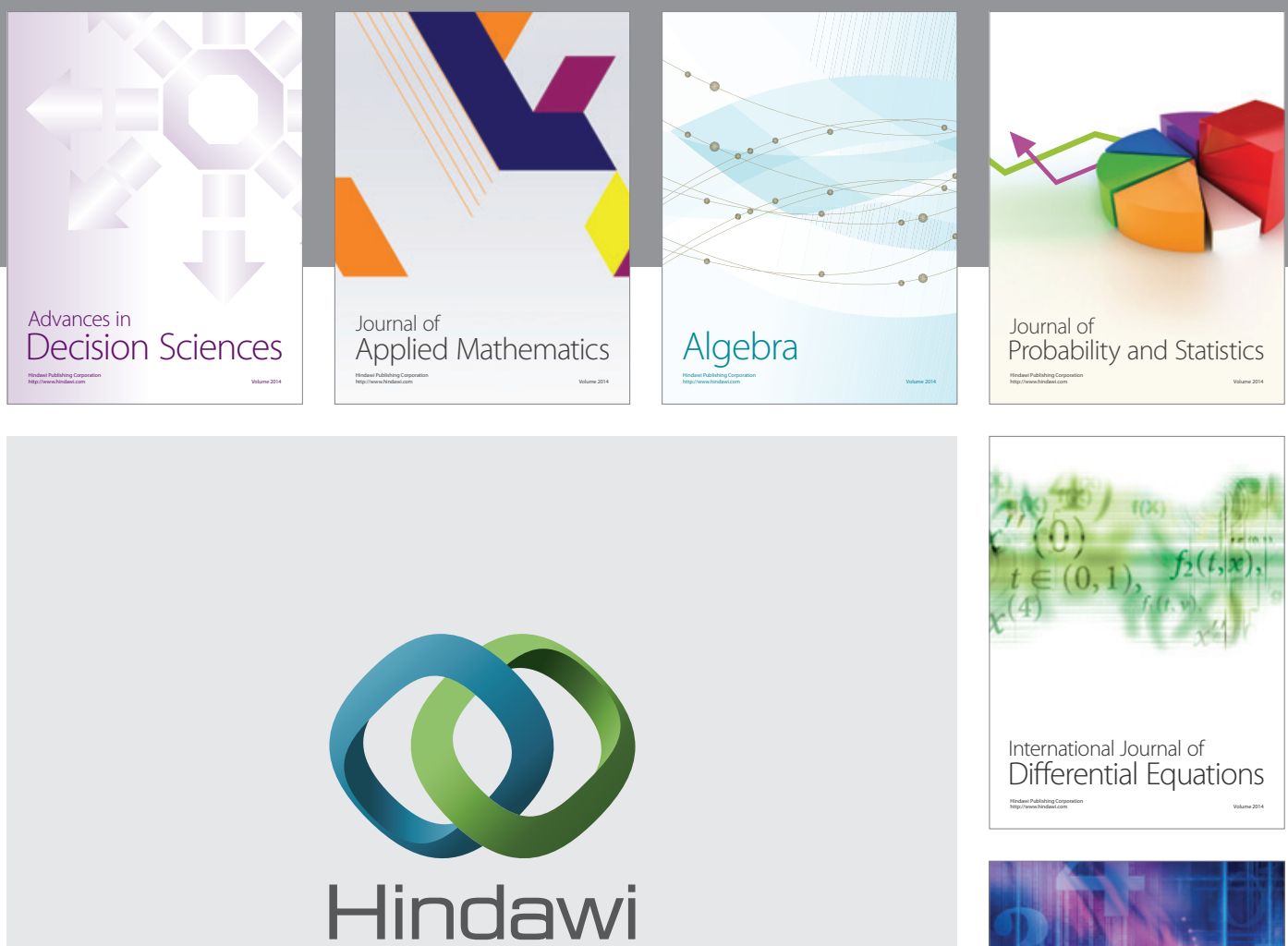

Submit your manuscripts at http://www.hindawi.com
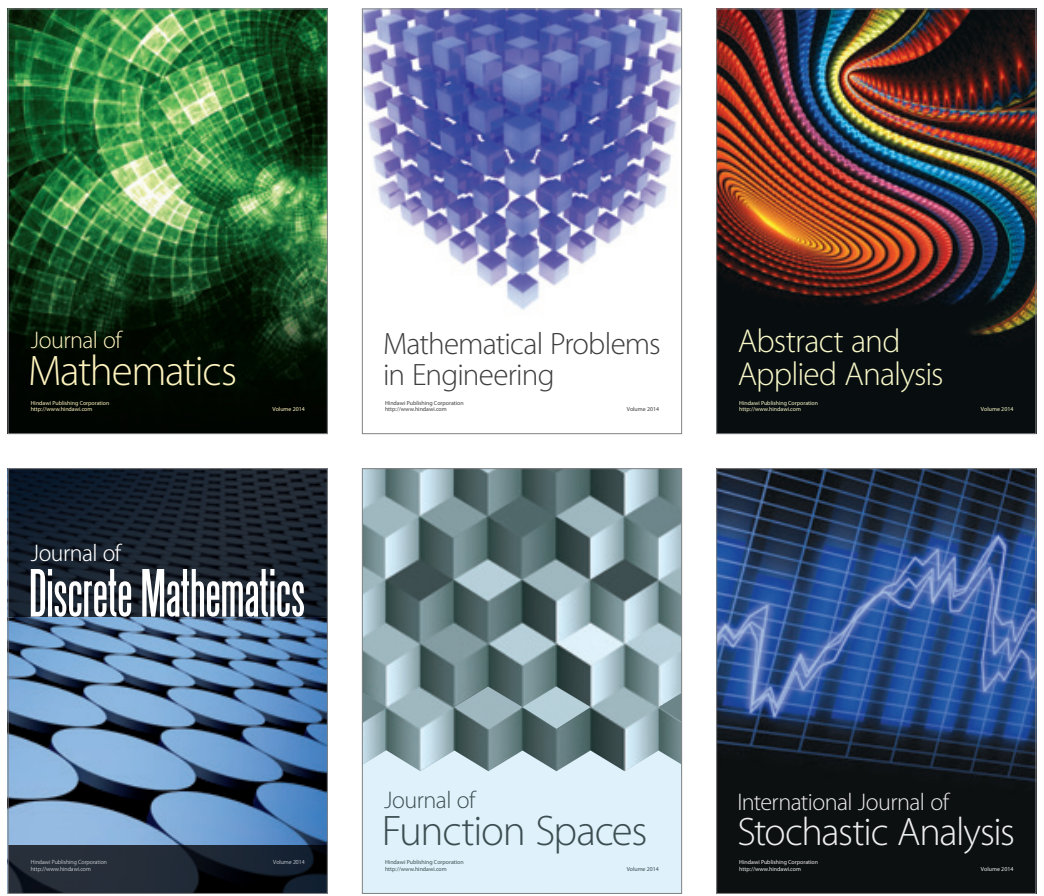

Journal of

Function Spaces

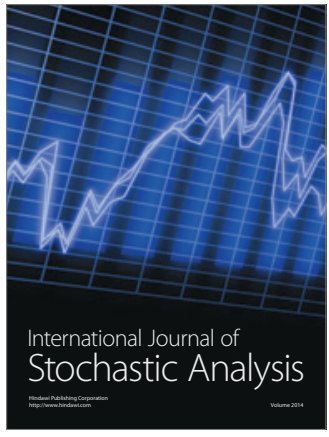

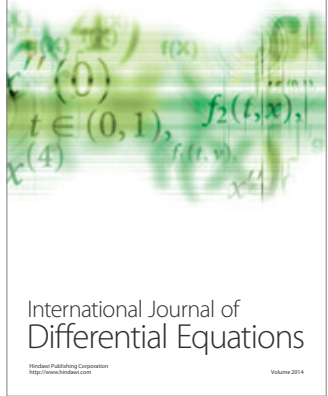
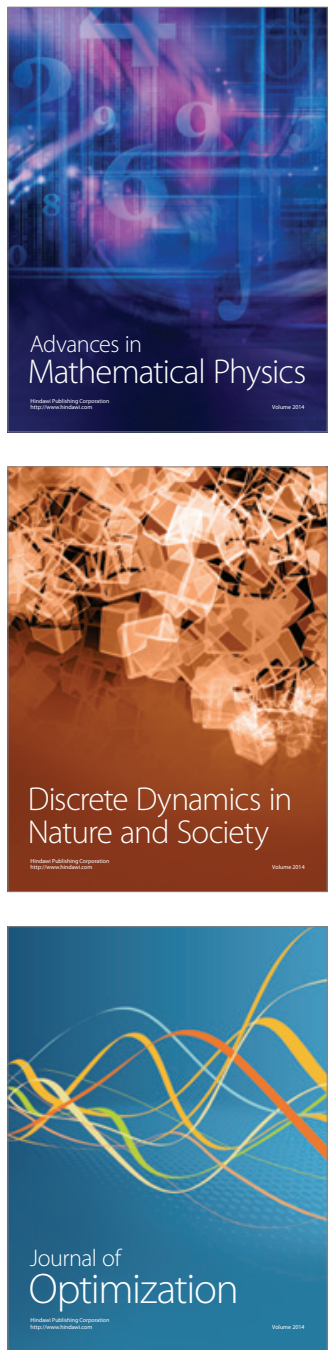